Rajendram $\mathrm{R}^{1,2^{*}}$

\title{
Acquired Long QT Syndrome: A Review of the Literature
}

${ }^{1}$ Consultant, Department of Medicine, King Abdulaziz Medical City, Ministry of National Guard - Health Affairs, Riyadh, Saudi Arabia

${ }^{2}$ Joint Appointment Assistant Professor of Medicine, College of Medicine, King Saud bin Abdulaziz University of Health Sciences, Riyadh, Saudi Arabia

Corresponding Author: Rajkumar Rajendram, AKC BSc (Hons) MBBS (Dist) MRCP (UK) EDIC FRCP Edin FRCP Lond

Address: Department of Medicine, King Abdulaziz Medical City, Ministry of National Guard - Health Affairs, Riyadh, Saudi Arabia; E-mail: rajkumarrajendram@doctors.org.uk

Received date: 29 January 2020; Accepted date: 19 February 2020; Published date: 27 February 2020

Citation: Rajendram R. Acquired Long QT Syndrome: A Review of the Literature. Asp Biomed Clin Case Rep. 2020 Feb 27;3(1):67-70.

Copyright (C) 2020 Rajendram R. This is an open-access article distributed under the Creative Commons Attribution License, which permits unrestricted use, distribution, and reproduction in any medium provided the original work is properly cited.

\begin{abstract}
The QT interval represents the duration of ventricular depolarization and repolarization. It is measured from the beginning of the QRS complex to the end of the T wave. Prolongation of the QT interval may be congenital or acquired. This increases the risk of polymorphic ventricular tachycardia (i.e torsades de pointes) and cardiac arrest. To increase the awareness of this life-threatening phenomenon I outline an illustrative case in which acquired prolongation of the QT interval due to electrolyte derangement and administration of ciprofloxacin resulted in cardiac arrest due to torsade de pointes. Management of a patient with a long QT syndrome includes Immediate cessation of drugs that prolong the QT interval; cardiac monitoring, serial 12 lead ECGs and transthoracic echocardiography; measurement of serum electrolytes; intravenous potassium replacement; intravenous magnesium replacement; beta-blockade. Causes of acquired prolongation of the QT interval are common in critically ill patients. It is important to recognize this and consider screening with 12 lead ECG to reduce the risk of life-threatening ventricular arrhythmias.
\end{abstract}

\section{Keywords}

Acquired Long QT Syndrome; Polymorphic Ventricular Tachycardia; Critical Illness; Electrolyte Derangement; Quinolone

\section{Introduction}

Prolongation of the QT interval may be congenital or acquired [1]. Congenital long QT syndromes result from mutations in genes encoding for the sodium and potassium channels in cardiac cells $[1,2]$. Delayed closure of the sodium channel results in prolongation of inward current while potassium channel mutations delay repolarization [1-3]. Several medications can prolong the QT interval by affecting cardiac myocyte ion channel function $\left[1,2,4^{-6}\right]$. Patients with drugassociated prolongation of the QT interval may also have mutations in ion channel genes [4]. Patients with acquired long QT syndrome may be thought to have self-terminating VT or VF unless the characteristic morphology of torsades de pointes and the QT prolongation is recognized [6]. Accurate diagnosis is essential as empirical antiarrhythmic therapy may worsen the arrhythmia through further 

27;3(1):67-70.

\section{Review Article}

lengthening of the QT interval [1-3;6,7].

\section{Illustrative Case}

A 30-year-old woman presented with a 3-day history of fever, dysuria, left flank pain, nausea, and vomiting. She was diagnosed with pyelonephritis and admitted for treatment with intravenous ciprofloxacin and metoclopramide.

Five days after re-admission a nurse witnessed the patient having a seizure whilst in bed. This was followed by cardiorespiratory arrest, and cardiopulmonary resuscitation (CPR) was started. The initial cardiac rhythm was treated as pulseless polymorphic ventricular tachycardia (VT; torsades de pointes).

Defibrillation resulted in sinus rhythm and spontaneous circulation (ROSC) and respiratory effort were restored after two minutes of CPR. Although the patient was initially drowsy, physical examination was otherwise unremarkable. The chest radiograph was also normal. However, a 12 lead electrocardiograph (ECG) performed immediately after ROSC revealed marked prolongation of the corrected QT interval (QTc $550 \mathrm{~ms}$ ). The initial ECG recorded on admission was normal (QTc $380 \mathrm{~ms}$ ).

Blood taken during CPR revealed: Sodium 138 $\mathrm{mmol} / \mathrm{L}$, potassium $2.5 \mathrm{mmol} / \mathrm{L}$, urea $2.7 \mathrm{mmol} / \mathrm{L}$, creatinine $75 \mathrm{mmol} / \mathrm{L}$, magnesium $0.45 \mathrm{mmol} / \mathrm{L}$, glucose $7.8 \mathrm{mmol} / \mathrm{L}$, haemoglobin $11 \mathrm{~g} / \mathrm{dL}$, white cell count $15 \times 109 / \mathrm{L}$, platelets $250 \times 1012 / \mathrm{L}$ and $\mathrm{C}$ reactive protein $250 \mathrm{mg} / \mathrm{dL}$.

The patient was transferred to the intensive care unit. Ciprofloxacin was stopped and replaced with meropenem. Metoclopramide was also stopped. Correction of magnesium to $1.0 \mathrm{mmol} / \mathrm{L}$, correction of the potassium to $4.5 \mathrm{mmol} / \mathrm{L}$ and administration of metoprolol over the next 24 hours reduced the QTc to $450 \mathrm{~ms}$. The patient was then transferred to the coronary care unit with no neurological deficit. An echocardiogram suggested mild anterior septal hypokinesis. However, coronary angiography and cardiac MRI were normal.
The QTc returned to the pre-arrest duration of 0.38 seconds two days after electrolyte replacement, stopping the metoclopramide and changing ciprofloxacin to meropenem. There were no further convulsions or arrhythmias and the patient was discharged home.

\section{Discussion}

The QT interval represents the duration of ventricular depolarization and repolarization [3]. It is measured on the surface ECG from the beginning of the QRS complex to the end of the $\mathrm{T}$ wave $[3,6]$. At least 3-4 cardiac cycles should be used to derive a mean value $[3,6]$. This mean value of the measured QT interval must then be corrected for heart rate $[3,6]$. Heart rate corrected QT Intervals (QTc) $>0.44 \mathrm{~s}$ are abnormal $[3,6]$.

The QT interval represents the summation of the action potentials (AP) of ventricular myocytes. The AP results from the flow of ions through channels across a cell membrane. If changes in ion concentrations or myocyte channel function increase the inward current or decrease the outward current, AP duration is increased and, the QT interval is prolonged.

Prolongation of the QT interval may be congenital or acquired [1]. In the present illustrative case prolongation of the QT interval was caused by the low serum potassium, low serum magnesium and ciprofloxacin.

Congenital long QT syndromes result from mutations in genes encoding for the sodium and potassium channels (IKr and IKs) in cardiac cells [13]. Delayed closure of the sodium channel results in prolongation of inward current while potassium channel mutations delay repolarisation $[2,3]$. Both mechanisms result in prolongation of the QT interval and increased disposition to early after depolarisations and polymorphic VT (torsade de pointes), often preceded by an increase in sinus rate [3].

A variety of congenital syndromes have been described including Jervell-Lange-Nielsen, an 
autosomal recessive condition associated with neural deafness; and Romano Ward, an autosomal dominant condition without deafness $[1,2]$. To date, more than 250 different mutations in seven genes have been identified as causing LQTS. The prevalence of long QT syndrome mutation carriers is therefore difficult to determine but estimates of up to 1 in 1000 have been suggested $[1,2]$.

Acquired LQTS is almost always due to blockage of the inward potassium rectifier (IKr) channel [8]. This channel is also known as the 'ether a go go' (hERG) channel. It conducts a rapid delayed rectifier potassium current (Ikr). This current occurs during phase 3 of the repolarization of the cardiac AP [8]. It has been proposed that patients with drug associated prolongation of the QT interval may also have mutations in ion channel genes [4].

Several medications can prolong the QT interval by affecting cardiac myocyte ion channel function $\left[1,2,4^{-}\right.$ 8]. A list of these medications is available at QTdrugs.org. However, drug-induced LQTS is not predictable. The molecular action of a drug does not always correlate with the clinical effect in any given patient. So, it is important to measure the QT interval of critically ill patients daily if they are being treated with any medication that can prolong the QT interval.

Antiarrhythmic medications are the most common cause of drug-induced torsade de pointes [8]. Vaughan Williams class IA agents (quinidine, procainamide and disopyramide) block both sodium and potassium channels. They can cause torsade de pointes even at subtherapeutic doses [8].

Vaughan Williams Class III agents are potent IKr blockers. Dofetilide, ibutilide and sotalol are associated with the highest risk of torsade de pointes [8]. Their effect is maximum at low heart rates. This characteristic is described as reverse use dependency [8]. Amiodarone is associated with the lowest risk [9]. Amiodarone decreases myocardial QT dispersion, lacks reverse use dependency, and blocks both L-type calcium channels and beta receptors [8]. As a result of these unique characteristics, amiodarone rarely causes torsade de pointes despite prolonging the QT interval.

Torsade de pointes in patients on antiarrhythmic therapy is often precipitated by hypokalaemia or hypomagnesaemia. So it is important to detect and treat electrolyte derangements in these patients.

Individual paroxysms of torsades de pointes are usually self-limiting; but if persistent, cardiac arrest will occur and defibrillation is necessary [3,6]. Episodes of torsades de pointes may be associated with sympathetic stimulation including exercise and fear and cause syncope and sometimes convulsion. Seizures caused by global cerebral ischaemia due to reduced cardiac output may be clinically indistinguishable from those caused by epilepsy $[6,7]$. As a result, patients may be misdiagnosed as This arrhythmia may also be misdiagnosed as selfterminating VT or VF unless the characteristic morphology of torsades de pointes and the QT interval prolongation is recognised [7]. Accurate diagnosis is essential as empirical antiarrhythmic therapy may worsen the arrhythmia by further lengthening the QT interval [5,7].

Causes of acquired prolongation of the QT interval are common in critically ill patients [7]. It is important to recognize this and consider screening with 12 lead ECG to reduce the risk of life-threatening ventricular arrhythmias [7].

In acquired long QT syndrome, any predisposing drugs should be discontinued and electrolyte disturbance, particularly hypokalaemia and hypomagnesaemia should be corrected [1,5-7]. Intravenous magnesium is safe and effective. Since the arrhythmia is associated with bradycardia and pauses, the heart rate should be increased, if necessary, through overdrive pacing or isoprenaline infusion. This should be done with caution in patients with acute ischaemia $[1,7]$. High dose beta blockade is effective in the congenital long QT syndrome although pacemaker or defibrillator implantation may be required in resistant cases $[1,2,6]$. A thorough drug history should be taken in cases of unexplained prolonged QT interval $[5,7]$ and the patient should be asked about any previous episodes of syncope or 

27;3(1):67-70.

\section{Review Article}

seizures or family history of such events [7].

\section{Conflicts of Interest}

None

\section{Sources of Funding}

None

\section{References}

[1] Chiang CE. Congenital and acquired long QT syndrome. Current concepts and management. Cardiol Rev. 2004 Jul-Aug;12(4):222-34. [PMID: 15191637]

[2] Collins KK, Van Hare GF. Advances in congenital long QT syndrome. Curr Opin Pediatr. 2006 Oct 1;18:497-502.

[3] Lankipalli RS, Zhu T, Guo D, Yan GX. Mechanisms underlying arrhythmogenesis in long QT syndrome. J Electrocardiol. 2005 Oct;38(4 Suppl):69-73.

[4] Yang P, Kanki H, Drolet B, Yang T, Wei J, Viswanathan PC, Hohnloser SH, Shimizu W, Schwartz PJ, Stanton M, Murray KT. Allelic variants in long-QT disease genes in patients with drugassociated torsades de pointes. Circulation. $2002 \mathrm{Apr}$ 23;105(16):1943-48.

[5] Owens RC Jr, Nolin TD. Antimicrobial-associated QT interval prolongation: pointes of interest. Clin Infect Dis. 2006 Dec 15;43(12):1603-11. [PMID: 17109296]

[6] Rajendram R, Ehtisham J, Forfar C. Oxford Case Histories in Cardiology. Oxford University Press; 2011 Mar 31.

[7] Priori SG, Napolitano C, Schwartz PJ. Low penetrance in the long-QT syndrome: clinical impact. Circulation. 1999 Feb 2;99(4):529-33.

[8] Nachimuthu S, Assar MD, Schussler JM. Druginduced QT interval prolongation: mechanisms and clinical management. Ther Adv Drug Saf. 2012 Oct;3(5):241-53. [PMID: 25083239]

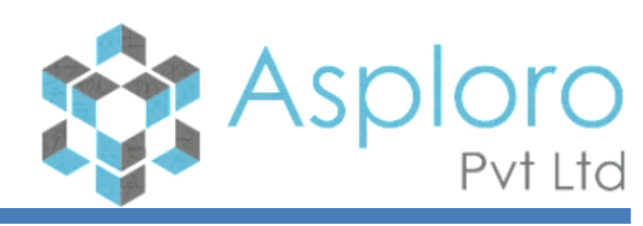

Keywords: Acquired Long QT Syndrome; Polymorphic Ventricular Tachycardia; Critical Illness; Electrolyte Derangement; Quinolone 